\title{
Corporación Andina de Fomento. Integridad en las Políticas Públicas: Claves para prevenir la corrupción. Bogotá, 2019.
}

De manera bastante pertinente, la Corporación Andina de Fomento ha tenido a bien publicar un acucioso reporte sobre el impacto de la corrupción en los gobiernos de América.

Su objetivo es brindar un análisis sobre el avance en el desarrollo de las políticas públicas anticorrupción y dar luces sobre los principales desafíos que enfrentan actualmente. De esta forma, se han identificado las líneas de acción más importantes emprendidas dentro y fuera de América, los aprendizajes obtenidos y los aportes de la literatura especializada.

El referido informe incluye gráficos comparativos y cuadros con datos regionales que brindan solidez a cada uno de los planteamientos ofrecidos. Podemos encontrar así información sobre la percepción ciudadana y empresarial sobre la corrupción, percepción entre corrupción y confianza, tiempos para realizar trámites, castigos judiciales a la corrupción, leyes de acceso a la información, sectores vulnerables a la corrupción, entre otros.

Una de las primeras conclusiones es la cuantificación que el estudio hace sobre el costo de la corrupción a la sociedad en términos económicos así como de bienestar y afectación al desarrollo. De ahí que se realice una primera correlación entre corrupción y desigualdad socioeconómica, disminución del crecimiento económico, inversión, y pérdida de credibilidad en las instituciones públicas.

De la misma forma, el reporte ofrece una definición de corrupción más amplia que la meramente legal a fin de abarcar las distintas modalidades que la ley no incluye. Vale decir, evita interpretar la corrupción exclusivamente como un tipo penal, para repensarla como un factor más grande de afectación al desarrollo del gobierno y al ejercicio de las políticas públicas.

En consecuencia, esta publicación hace mención a los arreglos institucionales como aquellas fórmulas que pueden reducir las posibilidades del ejercicio de prácticas de corrupción. Son cuatro las categorías en las que han sido agrupadas: reglas y control de la función pública, mecanismos de entrada a la burocracia y a la política, transparencia y monitoreo ciudadano, y la gobernanza de los intereses privados.

El primer aspecto sobre las las reglas y control de la función pública, propone determinar claramente los criterios para el ejercicio de esta de manera que se eviten subjetividades, por lo que su diseño debe ser muy cuidadoso a fin de evitar más medidas burocráticas. De igual forma, el control de fiscalización debe efectuarse durante todo el funcionamiento de la gestión (antes, durante y después) y se debe el fortalecer el mecanismo de sanción judicial.

Una segunda categoría abordada está relacionada a los mecanismos de entrada a la burocracia y a la política. El reporte plantea como principios la transparencia y la 
meritocracia y por consiguiente, la permanente comunicación de los logros obtenidos en cada gestión que facilitará la rendicion de cuentas y por ende el aumento de confianza de la ciudadanía. Estas reformas pueden implicar modificar las leyes de conformación de los partidos políticos, determinar nuevas reglas de financiamiento político, fortalecer los tribunales electorales, establecer procesos de reclutamiento a la función pública imparciales y fortalecimiento de la profesionalización del servicio civil.

El tercer punto analizado es la transparencia y el monitoreo ciudadano, que significa contar con información sobre quienes gestionan los recursos del Estado, sensibilizar a la población sobre la identificación de actos de corrupción, y generar canales de denuncia y reclamo. Para esto, resulta fundamental la promoción más amplia de la libertad de prensa y altos niveles de cobertura mediática.

Por último, la gobernanza de los intereses privados es un punto especialmente sensible puesto que significa hacer corresponsable al sector privado en los actos de corrupción. No basta -señala el estudio- con crear y aplicar normas e instrumentos legales, resulta necesario el fortalecimiento de los órganos de fiscalización e investigación y rediseñar los mecanismos de relacionamiento entre Estado y sector privado a fin de promover su integridad. Otro plano no menos importante es la gobernanza de las empresas estatales y sus procesos de toma de decisiones a nivel contractual, elección de funcionarios, obtención de ingresos, etc.

Este reporte termina con una propuesta para la implementación de una reforma bajo tres ejes: sistemas políticos contestatables, coordinación ciudadana y un líder creíble.

Otra conclusión que queremos resaltar es la puesta en valor de las herramientas digitales y el desarrollo del gobierno electrónico en la reducción de la corrupción en todas las categorías mencionadas.

Particularmente, consideramos que el principal mensaje del informe es el de demostrar que la prevención de la corrupción no se reduce a medidas cortoplacistas o aisladas sino, por el contrario, a la reforma de instituciones y mecanismos tanto del gobierno como del sector privado. Si se desea acceder a una mirada moderna y regional de la problemática, esta publicación representa un diagnóstico actualizado y una propuesta sumamente valiosa. 